\title{
Accumulation de la protéine soluble (PR 1a) dans les interactions Nicotiana tabacum/ Phytophthora ssp
}

\author{
N Maïa, L Cardin * \\ INRA, station de Botanique et de Pathologie végétale, villa Thuret, BP 2078, F06606 Antibes cedex, France
}

(Reçu le 15 mai 1992; accepté le 25 février 1993)

\begin{abstract}
Résumé - Le but de ce travail était de comparer les cinétiques d'accumulation de PR 1a, dans les différents niveaux d'interactions entre 4 génotypes de Nicotiana tabacum et 5 isolats de Phytophthora sp. Nous avons dans un premier temps vérifié sur gel de polyacrylamide que la protéine PR1a était majoritaire parmi les protéines de type PR dans les 4 génotypes. La quantification de la protéine PR 1a a été réalisée par la méthode immunoenzymatique DAS-Elisa, à partir d'extraits séparés des 2 feuilles situées immédiatement après le point d'inoculation de la tige. Les isolats de Phytophthora parasitica var nicotianae (NIC 181, NIC 183, NIC 310) agent du black shank sont seuls compatibles avec le $N$ tabacum. Une incompatibilité spécifique s'établit entre les variétés possédant le gène R1 (lignée d'Helgeson 46-8) et la race physiologique commune ro du champignon parasite. Cette résistance est surmontée par l'isolat de race $r 1$ (NIC 181). Certaines variétés, comme Escambray, possèdent une tolérance ou résistance non spécifique aux isolats de race 0 et 1 . Les variétés Xanthi nc et la lignée 49-10 d'Helgeson sont sensibles aux 2 races. Enfin l'incompatibilité générale (résistance "plante non hôte") peut être observée entre ces 4 génotypes et les 2 espèces étudiées de Phytophthora : $P$ cryptogea (CRY 52) et $P$ parasitica sensu lato (PAR 44) productrices d'élicitine (E+). Les 4 variétés ou lignées étudiées renfermaient avant décapitation de la tige et inoculation des quantités variables de $P R$ 1a $(0,03 \mu \mathrm{g} / \mathrm{gMF}$ à $6,6 \mu \mathrm{g} / \mathrm{gMF})$, celles-ci non corrélées à la résistance générale. La réaction de résistance de la lignée 46-8 (R1) à la race physiologique r0, n'a pas été marquée par une accumulation de PR 1a. Dans les interactions compatibles, le flétrissement du limbe foliaire, envahi par le parasite, s'accompagne dans tous les cas d'une accumulation importante de PR 1a (pouvant atteindre plus de $700 \mu \mathrm{g} / \mathrm{gMF}$ pour Escambray à J7). En ce qui concerne les interactions incompatibles de type plante «non hôte», l'interprétation des cinétiques observées est plus complexe. Une induction précoce de PR 1a à J1 pourrait être liée à l'action des éliciteurs produits par les espèces $P$ cryptogea et $P$ parasitica; l'accumulation plus tardive est beaucoup plus élevée entre $\mathrm{J} 4$ et $\mathrm{J} 7$ serait la conséquence indirecte des nécroses induites par la seule souche nécrosante $P$ cryptogea. Chez Xanthi nc, elle n'a pas été observée dans les interactions avec $P$ parasitica sensu lato qui n'a pas provoqué de nécrose à distance. Toute réaction nécrotique à distance ou envahissement des limbes s'accompagne d'une accumulation de PR 1a, mais nous n'avons pu mettre en évidence de relation directe entre la résistance et la synthèse de PR 1a.
\end{abstract}

\section{Nicotiana tabacum = tabac $/$ Phytophthora / PR 1a / Elisa}

Summary - Accumulation of pathogenesis-related protein (PR 1a) in Nicotiana tabacum/Phytophthora interactions. The aim of this study was to compare the kinetics of PR 1a accumulation in different types of interaction between 4 genotypes of $\mathrm{N}$ tabacum and 5 isolates of Phytophthora ssp. It was first shown by PAGE that PR 1a was predominantly induced in the 4 genotypes. The quantification of $P R$ 1a induced in the 2 leaves under the inoculation point was performed by an immunoenzymatic technique (DAS-ELISA). P parasitica var nicotianae (NIC) isolates are the only ones compatible with $\mathrm{N}$ tabacum. Specific incompatibility (vertical resistance) controlled by the gene $R 1$ occurs between 46-8 Helgeson's line and isolates NIC 183, NIC 310 race ro, whereas isolate NIC 181 race r1 overcomes it. The variety Xanthi nc and Hegelson's line 49-10 are susceptible to both races. General incompatibility (non-host resistance) is observed between all $\mathrm{N}$ tabacum genotypes and isolates of Phytophthora ssp other than $\mathrm{P}$ parasitica var nicotianae, for instance $P$ cryptogea (CRY 52) and P parasitica sensu lato (PAR 44). It has been demonstrated that incompatible species are more or less able to induce foliar necrosis without colonization and that their culture filtrates contain a protein elicitor (elicitin) identified respectively as cryptogein and parasiticein. Healthy plants of the 4 genotypes contain different amounts of PR 1a, ranging from $0.03 \mu \mathrm{g} / \mathrm{gFW}$ in Xanthi nc to $6.6 \mu \mathrm{g} / \mathrm{gFW}$ in Escambray. However, this level of PR 1a is not correlated with varietal resistance, either specific or nonspecific. The resistance process in the specifically incompatible interaction 46-8 (R1)/NIC 183 race ro is not associated with a noticeable increase of PR 1a in the leaves (table $\mathrm{IV}$ ). In the compatible interactions, petiole colonization and foliar wilt are followed by a

* Correspondance et tirés à part 
high level of PR la accumulation in Escambray reaching $>700 \mu \mathrm{g} / \mathrm{gFW}$ (table III). In the incompatible interactions, interpretation of the accumulation kinetics is more complex: early induction on the first day after inoculation could be directly stimulated by elicitins. This response depends on the varietal genotype: only Xanthi nc responds to parasiticein. Afterwards, a higher amount of PR la found on the fourth and seventh days in 46-8 and 49-10 lines inoculated with CRY 52 could be directly related to foliar necrosis; this peak is apparently absent in the same genotypes inoculated by the non-necrotic isolate PAR 44. In conclusion, from our results it seems that all necrotic reactions induced by incompatible Phytophthora species or foliar invasion by compatible strain release a large amount of PR 1a. On the other hand, no direct relation between any resistance state and PR ta accumulation has been clearly established.

Nicotiana tabacum $=$ tobacco $/$ Phytophthora $/$ PR 1a $/$ Elisa

\section{INTRODUCTION}

La maladie du black-shank des cultures de tabac est provoquée par les isolats de Phytophthora parasitica var nicotianae (Ppn). Selon les concepts des relations hôte-parasite développés par Heath (1981), "l'incompatibilité générale" (résistance plante non-hôte) intervient entre le tabac et toutes les espèces ou variétés de Phytophthora autres que les isolats de Ppn, seuls compatibles. Un gène $R 1$ d'incompatibilité spécifique (résistance verticale) aux isolats de la race commune $P p n$ race ro a été introduit par hybridation interspécifique entre $N$ tabacum et $N$ plumbaginifolia par Helgeson (1976) dans la lignée 46-8 de type Virginie. Cette lignée 46-8 (R1) est donc résistante aux isolats $P p n$, race ro, mais sensible aux isolats de race $r 1$ capables de contourner cette résistance. Enfin, différentes variétés possèdent une incompatibilité non spécifique (résistance horizontale) aux isolats $P p n$ race ro et $r 1$.

L'expression de l'incompatibilité générale (plante non-hôte) entre l'espèce cultivée Nicotiana tabacum var Xanthi nc ou var Samsun, et les isolats de Phytophthora ssp a été décrite par Bonnet et al (1986). Avec les isolats incompatibles, inoculés sur tiges de tabac décapités à l'état végétatif, l'envahissement caulinaire est faible ou nul, alors que l'on observe une réaction qualifiée de "type hypersensible" caractérisée par des nécroses foliaires à distance. Cette réaction nécrotique est plus ou moins marquée selon les espèces du genre Phytophthora: ainsi, l'espèce $P$ cryptogea est fortement nécrosante, l'espèce $P$ parasitica faiblement nécrosante.

La même réaction nécrotique est déclenchée par une protéine de $10 \mathrm{kDa}$ excrétée dans le filtrat de culture des espèces incompatibles. La séquence de cette protéine est caractéristique de chaque espèce: cryptogéine de $P$ cryptogea (Ricci et al, 1989), parasiticéine de $P$ parasitica (Bruneteau, communication personnelle). Cette famille de protéines dites "élicitines" possède des propriétés d'éliciteurs de résistance à l'égard des souches compatibles. En première analyse, les isolats compatibles très agressifs de $P p n$ originaires des États-Unis ou de Cuba en sont dépourvus, mais des isolats peu agressifs de blackshank d'origine australienne en produisent (Bonnet, communication personnelle).

La réaction de type «hypersensible» de l'interaction plante "non hôte" est toujours marquée par un pic d'accumulation de la protéine soluble PR 1a, $4 \mathrm{j}$ après inoculation par des isolats incompatibles nécrosants ou peu nécrosants, ou par l'apport de leur filtrat de culture sur les tiges décapitées de tabacs Xanthi nc ou Samsun (Bonnet et al, 1986). Dans les interactions compatibles, une faible accumulation de PR 1a est décelable entre les $8^{e}$ et $15^{e}$ j qui suivent l'inoculation, dans les feuilles situées en avant du front d'envahissement caulinaire. Les auteurs posent la question des relations entre réaction nécrotique et synthèse de PR 1a. Parmi les 25 protéines de type $P R$ décrites dans la littérature (Stintzi et al, 1990), la PR 1a, dont la fonction biologique n'est pas connue, est dans le genre Nicotiana généralement la plus abondante. Son accumulation a été signalée dans un grand nombre d'interactions hôte-parasite: bactéries, champignons, virus, viroïdes. Elle est induite par certains composés abiotiques dans des situations de stress ou des états physiologiques particuliers (Kauffmann, 1988).

C'est pourquoi nous l'avons retenue comme «marqueur» dans les différents types de relation entre le tabac et le phytophthora, et nous avons comparé les cinétiques de son accumulation dans les niveaux d'interactions précédemment décrits :

- incompatibilité générale avec les espèces : $P$ cryptogea fortement nécrosant; $P$ parasitica sensu lato faiblement nécrosant ;

- incompatibilité spécifique gène $\mathrm{R} 1 / P p n$ race $\mathrm{r} 0$;

- incompatibilité non spécifique ;

- compatibilité. 


\section{MATÉRIEL ET MÉTHODES}

\section{Matériel biologique}

\section{Variétés de tabac}

Nous avons inoculé 4 génotypes de tabac. La variété Xanthi nc est sensible aux différents isolats de Ppn. La lignée 46-8 possède le gène de résistance spécifique $R 1$ à la race physiologique commune ro, elle est sensible aux isolats de race $r 1$, sa lignée isogénique 4910 est sensible aux isolats de $P p n$ ro et $r 1$; ces 2 lignées sont issues de l'hybridation interspécifique entre les espèces $N$ tabacum et $N$ plumbaginifolia (Helgeson et al, 1976). Dans le texte, par opposition à la lignée 46-8 R1, nous désignerons par R0 les 3 génotypes dépourvus de ce gène de résistance.

\section{Matériel fongique}

Isolats incompatibles : $P$ parasitica sensu lato (PAR 44) isolé de citrus faiblement nécrosant sur feuille de tabac, et $P$ cryptogea (CRY 52) isolé de Gerbera fortement nécrosant. Les deux isolats sont producteurs d'élicitine.

Isolats compatibles, agents du black-shank: $P$ parasitica var nicotianae (NIC 181) race $\mathrm{r1}$, NIC (183) race r0 ont été isolés de tabac aux États-Unis. L'isolat (NIC 310) isolé de tabac en Australie, produit dans son filtrat de culture une élicitine comparable à celle des souches incompatibles (Bonnet, communication personnelle).

\section{Système expérimental}

\section{Inoculations, symptômes et prélèvements}

Les plants de tabac sont cultivés en chambre climatisée : $23^{\circ} \mathrm{C}, 14 \mathrm{~h}$ d'éclairement à 16000 lux. Les tabacs sont décapités et on apporte sur la blessure un disque de culture mycélienne sur malt gélosé. Dans le tableau I, sont indiquées les différentes interactions étudiées. Nous avons inoculé des plants des variétés Xanthi nc et Escambray âgés de $60 \mathrm{j}$. Les plants des lignées 49-10 et 46-8, inoculés dans les 2 essais successif, étaient âgés de 73 j ou 80 j. Ces 2 lignées ont un allongement caulinaire plus lent; de ce fait le test sur tiges décapitées a été impossible à réaliser à $60 \mathrm{j}$.

On a noté à $\mathrm{J} 2$ la réaction nécrotique de type «hypersensible», en relevant le nombre de feuilles présentant des nécroses à distance.

À J2, J4, J7, pour l'ensemble des souches, on a mesuré en millimètres, l'envahissement caulinaire et éventuellement celui des pétioles. À ces mêmes dates, les feuilles $F 1$ et $F 2$ situées immédiatement sous la blessure, sont prélevées pour l'extraction de la protéine PR 1a. Chaque prélèvement a été fait sur un seul plant. Pour chacun des quatre génotypes, un prélèvement à J0 (avant décapitation) indique le taux constitutif de PR $1 \mathrm{a}$.

\section{Préparation des extraits et électrophorèse en PAGE}

Les feuilles $F 1$ de plusieurs plants des 4 génotypes ont été prélevées à $\mathrm{J} 0$ et $\mathrm{J} 7$ suite à l'inoculation par la souche CRY 52 inductrice de nécroses foliaires à distance. En référence, des feuilles de Xanthi nc présentant des lésions locales ont été récoltées $4 \mathrm{j}$ après inoculation par le virus de la mosaïque du tabac.

La préparation des extraits a été faite selon Abad et al (1986). Elle consiste à broyer au mortier les feuilles congelées dans un tampon de $84 \mathrm{mM}$ acide citrique et $32 \mathrm{mM}$ phosphate disodique à $\mathrm{pH} 2,8$. Après filtration sur étamine, le jus est clarifié par 2 centrifugations à 5000 puis $14000 \mathrm{~g}$ durant 10 et 20 min. On procède à une précipitation par adjonction au surnageant de $50 \%$ (poids/volume) de sulfate d'ammonium pendant $30 \mathrm{~min}$ à $4^{\circ} \mathrm{C} /$ Après centrifugation à $10000 \mathrm{~g}$ durant $20 \mathrm{~min}$, le culot est repris dans un tampon $10 \mathrm{mM}$ tris- $\mathrm{HCl}(\mathrm{pH} 8,0)$ puis dialysé abondamment contre ce même tampon à $4^{\circ} \mathrm{C}$ durant la nuit. La technique d'electrophorèse en gel de polyacrylamide est décrite par Abad et al (1986). L'electrophorèse sur gel de polyacryamide de $15 \%$ (plaque de $120 \times 85 \times 1 \mathrm{~mm}$ ) se fait en condition non dénaturante sous un voltage constant de $150 \vee$ durant $3 \mathrm{~h}$. Le dépôt de chaque échantillon correspond à l'équivalent de $0,1 \mathrm{~g}$ de MF. Les gels sont colorés dans une solution eau-méthanol-acide acétique (5-5-1) contenant $0,03 \%$ de bleu de Coomasie Brillant Blue R 250 . Après décoloration dans la même solution, sans colorant, les gels sont séchés.

\section{Extraction et dosage de la PR 1a}

On a dosé la PR 1a séparément dans les feuilles $F 1$ et F2 (de 1,5 à 8,6 gMF), par la méthode immunoenzymatique (DAS-Elisa) décrite pour cette même protéine par Abad et al (1986). En comparaison avec la technique de dosage par HPLC, Abad et al ( 1989) ont démontré qu'en dépit des parentés sérologiques entre les protéines acides de cette famille $(1 \mathrm{a}, 1 \mathrm{~b}, 1 \mathrm{c})$ la technique DAS-Elisa, permettait une quantification fiable de la PR 1a, sans que les PR 1b et PR 1c interviennent notablement dans le dosage.

La technique de dosage choisie a l'avantage de pouvoir traiter rapidement un nombre élevé d'échantillons à partir d'un extrait obtenu par simple broyage dans le même tampon que précédemment $(\mathrm{pH} 2,8)$ et centrifugation à faible vitesse. On établit une gamme de dilution très large des extraits (progression géométrique de raison 2), dans du tampon $10 \mathrm{mM}$ tris- $\mathrm{HCl}+$ $0,5 \%$ Tween 20 et $1 \%$ d'albumine de sérum bovin $(\mathrm{pH}$ 8,0 ). Ainsi, pour le test Elisa, des valeurs de densité optique se situeront dans la zone de dosage préala- 
Tableau I. Interactions étudiées entre Nicotiana tabacum et Phytophthora ssp.

Variété de tabac

Isolats de Phytophthora

\begin{tabular}{ccccc}
\hline CRY 52 & PAR 44 & NIC 310, ro & NIC 183, ro & NIC 181, r1 \\
$E_{+}$ & $E_{+}$ & $E_{+}$ & $E_{-}$ & $E_{-}$
\end{tabular}

Xanthi nc sensible aux races 0 et 1

Escambray tolérant aux races 0 et 1

49-10 sensible aux races 0 et 1

$46-8$ résistant à $r 0$ sensible à $r 1$

$\begin{array}{lll}\text { INE } & \text { ICG } & \text { C } \\ \text { INE } & \text { ICG } & \text { INE } \\ \text { ICG } & \text { ICG } & \text { INE } \\ \text { ICG } & \text { ICG } & \text { INE }\end{array}$

C

ICNS

$\mathrm{C}$

ICS
$\mathrm{C}$

ICNS

$\mathrm{C}$

C

C : compatibilité; ICS : incompatibilité spécifique (gène R1/race physiologique ro); ICNS : incompatibilité non spécifique (résistance horizontale); ICG : incompatibilité générale "plante non hôte»; INE : interaction non étudiée; E+ : isolats de Phytophthora producteurs d'élicitines; E- : isolats de Phytophtora non producteurs d'élicitines.

blement définie par une gamme étalon donnée avec de la protéine PR 1a pure (purification par HPLC).

Deux répétitions sont effectuées pour chaque dilution. Les valeurs de DO, mesurées à $405 \mathrm{~nm}$, sont enregistrées par un appareil Titrek Twinreader et traitées à l'aide d'un programme Flow-Laboratory en tenant compte des indications données par Cardin et al (1983).

La sensibilité de la technique est de $0,25 \mathrm{ng} / \mathrm{ml}$ de PR 1a avec une gamme de dosage comprise entre 0,5 et $64 \mathrm{ng} / \mathrm{ml}$. Les coefficients de variations intraessais et interessais étaient respectivement de l'ordre de $5 \%$ et de 15 à $20 \%$ (Abad et al, 1989).

\section{RÉSULTATS}

\section{Électrophorégramme en PAGE des 4 génotypes étudiés (fig 1)}

Avant de doser la PR 1a en Elisa dans les différentes interactions souches/variétés étudiées, nous avons comparé les électrophorégrammes obtenus en gel de polyacylamide (15\%) en conditions non dénaturantes des 4 génotypes. Cette étude a été faite sur des extraits de la feuille F1 de plants sains (JO) et de plants inoculés par l'isolat CRY 52 (J7). La feuille F1 des 4 génotypes inoculés présentait des nécroses foliaires. Nous avons pris comme référence un extrait de feuille de la variété Xanthi nc inoculé par du VMT.

Par cette technique, on ne décèle de PR que dans les plants inoculés par l'isolat CRY 52. En référence au témoin VMT, les génotypes Xanthi nc, $49-10$ et $46-8$ ont des profils sensiblement identiques avec la bande $\operatorname{Pr} 1$ a majoritaire; la variété Escambray présente également une bande $\operatorname{Pr} 1$ a majoritaire, ainsi qu'une bande surnuméraire située au dessus de cette dernière (fig 1). Après avoir découpé et élué cette bande, nous avons vérifié, par immuno-électrophorèse en gel d'agarose, qu'elle était reliée sérologiquement à la PR 1a (résultats non présentés).

\section{Teneur en PR 1a des plants non inoculés}

Les teneurs en PR 1a des plants non inoculés avant décapitation (J0) ou à $1 \mathrm{j}(\mathrm{J} 1), 2 \mathrm{j}(\mathrm{J} 2), 4 \mathrm{j}$ (J4) ou 7 j (J7) après la décapitation figurent dans le tableau II. Les plants des variétés Xanthi nc et Escambray étaient âgés de $60 \mathrm{j}$. Pour les lignées isogéniques dont l'élongation caulinaire

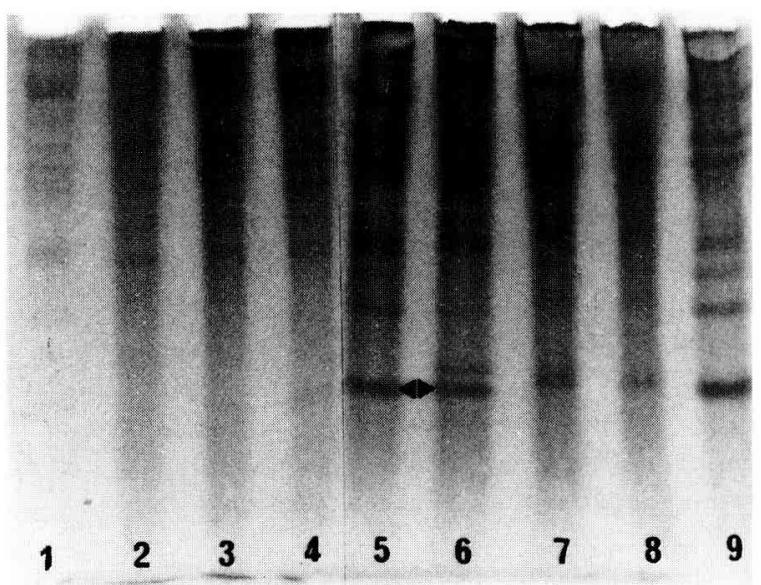

Fig 1. Électrophorégramme en PAGE $15 \%$ en conditions non dénaturantes d'extraits de feuilles de tabac. Piste 1, 2, 3, 4 : témoins non inoculés (JO) de Xanthi nc; Escambray; 49-10; 46-8. Piste 5, 6, 7, 8 : feuille $F 1$ (J7) après inoculation par CRY 52 de Xanthi nc; Escambray; 49-10; 46-8. Piste 9; feuille de Xanthi nc (J4) inoculé par du VMT. 
est plus lente, nous avons dû utiliser des plants plus âgés : 73 ou $80 \mathrm{j}$. Chaque dosage a été fait sur les feuilles 1 et 2, d'un seul plant: l'effet «individu» intravariétal n'a pu donc être apprécié. La variété Escambray, les lignée $49-10$ et $46-8$ renferment des teneurs en PR 1a sensiblement plus élevées que la variété Xanthi nc. Cette teneur augmente avec l'âge de la feuille et l'âge du plant. D'après les valeurs dosées dans les 7 jours qui ont suivi la décapitation, on observe une variation importante, sans que se dégage nettement une cinétique d'accumulation de PR 1a, propre à l'effet blessure.

\section{Symptômes observés après inoculation}

\section{Incompatibilité générale (tableau III)}

L'isolat de $P$ cryptogea, CRY 52 incompatible, n'envahit pas la tige des lignées 49-10 et 46-8. Dès le $2^{e} \mathrm{j}$ après l'inoculation, des réactions nécrotiques apparaissent sur les feuilles de rang 1 à 4 situées sous le point d'inoculation.

L'isolat $P$ parasitica 44 , inoculé sur les 4 génotypes, n'envahit que faiblement les tiges (10 à 18 $\mathrm{mm}$ ), sans provoquer de réactions nécrotiques à distance (fig 2).

\section{Incompatibilité spécifique}

Dans l'interaction d'incompatibilité spécifique entre la lignée (R1) 46-8 et les isolats de race physiologique $\mathrm{rO}$ : NIC 183 et NIC 310 , le champignon n'envahit pas la tige, et aucune réaction, de type nécrose foliaire à distance, n'est apparue (fig 2).

\section{Compatibilité}

Les isolats compatibles envahissent la tige et colonisent successivement les pétioles foliaires, entraînant le flétrissement du limbe. Sur les graphiques de la figure 2, sont représentées les courbes d'envahissement caulinaire pour les quatre génotypes étudiés ainsi que le nombre de pétioles envahis à $\mathrm{J} 7$ ([x] voir légende). Dans ce mode d'inoculation sur tige décapitée, la résistance horizontale (non-spécifique) de la variété Escambray se traduit par le fait que seul le pétiole de la première feuille est envahi à $\mathrm{J} 7$ par les souches compatibles. La lignée 46-8 (R1) se montre moins sensible à l'isolat NIC 181 race 1 que la lignée isogénique 49-10. Sur les trois génotypes Xanthi nc, Escambray et 49-10 dépourvus du gène R1, I'isolat NIC 310 , producteur d'élicitine $(E+)$, est moins agressif que les isolats (E-), NIC 181 et NIC 183.

Tableau II. Accumulation de PR 1 a exprimée en $\mu \mathrm{g} / \mathrm{gMF}$ dans les feuilles (F1) et (F2) de tabacs sains non décapités $(\mathrm{J} 0)$, ou $1 \mathrm{j}(\mathrm{J} 1), 2 \mathrm{j}(\mathrm{J} 2), 4 \mathrm{j}(\mathrm{J} 4)$ et $7 \mathrm{j}(\mathrm{J} 7)$, après décapitation sans inoculation.

\begin{tabular}{|c|c|c|c|c|c|c|c|}
\hline \multirow[t]{2}{*}{ Variétés } & \multirow{2}{*}{$\begin{array}{l}\hat{A} \text { ges des } \\
\text { plants (en j) }\end{array}$} & \multirow{2}{*}{$\begin{array}{l}\text { Niveau des } \\
\text { feuilles }\end{array}$} & \multicolumn{5}{|c|}{ Teneur en PR 1a ( $\mu \mathrm{g} / \mathrm{gMF})$} \\
\hline & & & Jo & $J 1$ & $\sqrt{2}$ & $J 4$ & $J 7$ \\
\hline Xanthi nc & 60 & $\begin{array}{l}1 \\
2\end{array}$ & $\begin{array}{l}0,03 \\
0,01\end{array}$ & $\begin{array}{l}0,01 \\
0,09\end{array}$ & $\begin{array}{l}0,02 \\
0,17\end{array}$ & $\begin{array}{l}0,04 \\
0,37\end{array}$ & $\begin{array}{l}0,03 \\
0,10\end{array}$ \\
\hline Escambray & 60 & $\begin{array}{l}1 \\
2\end{array}$ & $\begin{array}{r}6,60 \\
21,60\end{array}$ & $\begin{array}{l}2,5 \\
3,9\end{array}$ & $\begin{array}{l}12,9 \\
21,7\end{array}$ & $\begin{array}{r}5,40 \\
15,80\end{array}$ & $\begin{array}{l}3,70 \\
4,80\end{array}$ \\
\hline $49-10$ & 73 & $\begin{array}{l}1 \\
2\end{array}$ & $\begin{array}{l}0,84 \\
1,01\end{array}$ & $\begin{array}{l}6,23 \\
3,24\end{array}$ & $\begin{array}{l}1 \\
8\end{array}$ & $\begin{array}{r}26,90 \\
1,57\end{array}$ & $\begin{array}{l}4,34 \\
9,77\end{array}$ \\
\hline $46-8$ & 73 & $\begin{array}{l}1 \\
2\end{array}$ & $\begin{array}{l}0,01 \\
0,27\end{array}$ & $\begin{array}{l}0,29 \\
0,14\end{array}$ & $\begin{array}{l}1,73 \\
2,43\end{array}$ & $\begin{array}{r}2,18 \\
12,10\end{array}$ & $\begin{array}{r}3,54 \\
13,70\end{array}$ \\
\hline $49-10$ & 80 & $\begin{array}{l}1 \\
2\end{array}$ & $\begin{array}{l}1,42 \\
1,67\end{array}$ & $\begin{array}{l}2,01 \\
0,60\end{array}$ & $\begin{array}{l}2,23 \\
2,03\end{array}$ & $\begin{array}{l}0,69 \\
2,58\end{array}$ & $\begin{array}{l}4,67 \\
3,95\end{array}$ \\
\hline $46-8$ & 80 & $\begin{array}{l}1 \\
2\end{array}$ & $\begin{array}{r}3,66 \\
16,20\end{array}$ & $\begin{array}{l}5,41 \\
9,92\end{array}$ & $\begin{array}{l}7,73 \\
6,10\end{array}$ & $\begin{array}{r}0,44 \\
10,90\end{array}$ & $\begin{array}{l}12,20 \\
42,20\end{array}$ \\
\hline
\end{tabular}


Tableau III. Symptômes observés et accumulation de PR 1a en $\mu \mathrm{g} / \mathrm{gMF}$ dans la première feuille (F1) et dans la seconde feuille (F2) après inoculation des 4 génotypes de tabac par les souches incompatibles $P$ cryptogea (CRY 52) et P parasitica (PAR 44).

\begin{tabular}{|c|c|c|c|c|c|c|c|c|c|c|}
\hline \multirow[t]{2}{*}{ Isolats } & \multirow[t]{2}{*}{ Variétés } & \multirow{2}{*}{$\begin{array}{l}\text { Âges du } \\
\text { plant } \\
\text { (en j) }\end{array}$} & \multirow{2}{*}{$\begin{array}{l}\text { Longueur de } \\
\text { l'envahis- } \\
\text { sement } \\
\text { caulinaire } \\
\text { (en mm) }\end{array}$} & \multirow{2}{*}{$\begin{array}{l}\text { Feuilles } \\
\text { présentant des } \\
\text { nécroses (à } J 2)\end{array}$} & \multicolumn{6}{|c|}{ Teneur en PR 1a $(\mu g / g M F)$} \\
\hline & & & & & $\begin{array}{l}\text { Niveaux } \\
\text { Feuilles }\end{array}$ & JO & $J 1$ & $\sqrt{ } 2$ & J4 & $J 7$ \\
\hline \multirow{2}{*}{$\begin{array}{l}\text { P cryptogea } \\
\text { CRY } 52\end{array}$} & $49-10$ & 73 & 0 & F2 F3 F4 & $\begin{array}{l}F 1 \\
F 2\end{array}$ & $\begin{array}{l}0,8 \\
1\end{array}$ & $\begin{array}{r}13 \\
4\end{array}$ & $\begin{array}{l}19 \\
14\end{array}$ & $\begin{array}{l}243 \\
261\end{array}$ & $\begin{array}{l}381 \\
541\end{array}$ \\
\hline & $46-8$ & 73 & 0 & $F_{1} F_{2} F_{3} F_{4}$ & $\begin{array}{l}F 1 \\
F 2\end{array}$ & $\begin{array}{l}0,01 \\
0,2\end{array}$ & $\begin{array}{l}1,8 \\
1,6\end{array}$ & $\begin{array}{l}17 \\
12\end{array}$ & $\begin{array}{l}52 \\
43\end{array}$ & $\begin{array}{l}184 \\
174\end{array}$ \\
\hline \multirow{7}{*}{$\begin{array}{l}\text { P parasitica } \\
\text { PAR } 44\end{array}$} & Xanthi nc & 60 & 18 & \multirow{7}{*}{$\begin{array}{l}\text { Absence de } \\
\text { nécroses } \\
\text { foliaires }\end{array}$} & $\begin{array}{l}F 1 \\
F 2\end{array}$ & $\begin{array}{l}0,03 \\
0,01\end{array}$ & $\begin{array}{l}2,6 \\
8\end{array}$ & $\begin{array}{l}0,5 \\
0,7\end{array}$ & $\begin{array}{l}0,7 \\
4\end{array}$ & $\begin{array}{c}5 \\
13\end{array}$ \\
\hline & Escambray & 60 & 16 & & $\mathrm{~F} 1$ & 7 & 2,6 & 3 & 10 & 6 \\
\hline & & & & & $F 2$ & 22 & 5 & 5 & 6 & 11 \\
\hline & & & & & $F_{1}$ & 1,4 & 0,5 & 6 & 4 & 9 \\
\hline & $49-10$ & 80 & 10 & & $\mathrm{~F} 2$ & 1,6 & 1,5 & 3 & 11 & 7 \\
\hline & $46-8$ & 80 & 10 & & $F 1$ & 3,6 & 0,6 & 0,4 & 8 & 11 \\
\hline & & & & & $\mathrm{F} 2$ & 1,6 & 16 & 1,6 & 26 & 31 \\
\hline
\end{tabular}

\section{Cinétique d'accumulation de la PR $1 a$}

\section{Incompatibilité générale (tableau III)}

En réponse à l'isolat $P$ cryptogea 52 , la teneur en PR 1a s'élève dès $J 1$ dans les lignées 49-10 et $46-8$, mais les valeurs observées sont de l'ordre de celles des témoins sains décapités (tableau I). C'est à partir de J4 que les valeurs sont indiscutablement supérieures à celles relevées dans les témoins.

En réponse à l'isolat $P$ parasitica (PAR 44), seule la variété $X a n t h i n c$ réagit par une augmentation notable de PR 1a. Cette teneur passe de $0,03 \mu \mathrm{g} / \mathrm{gMF}$ dans les plants témoins non décapités à $8 \mu \mathrm{g} / \mathrm{gMF}$ à $\mathrm{J} 1$ dans la seconde feuille. Les 3 autres génotypes ne réagissent pas à l'inoculation de cet isolat incompatible, qui ne provoque pas de nécrose à distance. Leur taux constitutif de PR 1a plus élevé peut masquer la réponse à cet isolat.

\section{Incompatibilité spécifique (tableau IV)}

Cette incompatibilité $\mathrm{R} 1 / P p n$ race $\mathrm{r} 0$ intervient entre la lignée 46-8 (R1) et l'isolat NIC 183 de race physiologique ro incapable d'envahir la tige décapitée. On n'a pas décelé d'accumulation notable de PR 1a, dans les feuilles de la lignée 46-8 âgée de 73 ou 80 j inoculée par l'isolat NIC 183 race ro. Mais elle serait peut-être décelable dans les tissus de la tige plus proche du site d'interaction.

\section{Interactions compatibles (tableau V)}

Dans les interactions compatibles avec les isolats non producteurs d'élicitine NIC 181 et NIC 183, l'envahissement successif des pétioles des feuilles 1 et 2 entre $J 4$ et $J 7$, qui provoque le flétrissement du limbe foliaire, est marqué par une accumulation brutale de PR 1 a à J4 dans la première feuille, à $J 7$ dans la seconde feuille.

La cinétique d'accumulation est identique dans les 4 génotypes. À $\mathrm{J} 7$, dans la première feuille des 4 génotypes inoculés par NIC 181, on a relevé respectivement (tableau V): $108 \mu \mathrm{g} /$ g MF pour Xanthi nc, $711 \mu \mathrm{g} / \mathrm{gMF}$ pour Escambray, $403 \mu \mathrm{g} / \mathrm{gMF}$ et $261 \mu \mathrm{g} / \mathrm{gMF}$ pour $49-10$ et 46-8 inoculés par l'isolat 181. Les résultats sont comparables pour l'isolat NIC 183. Dans la seconde feuille, l'amplitude de l'accumulation est 


\section{SOUCHE 181}

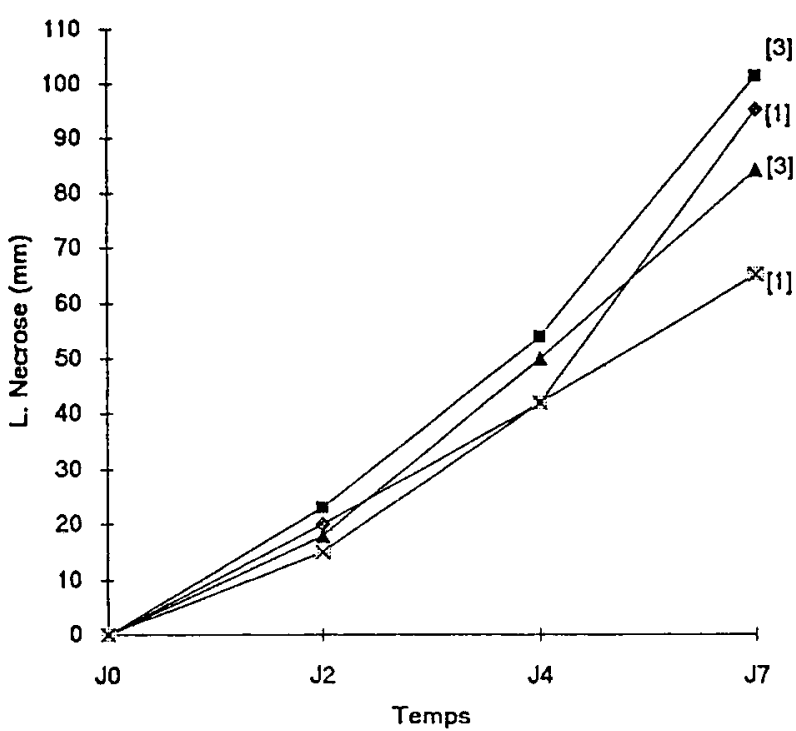

\section{SOUCHE 310}

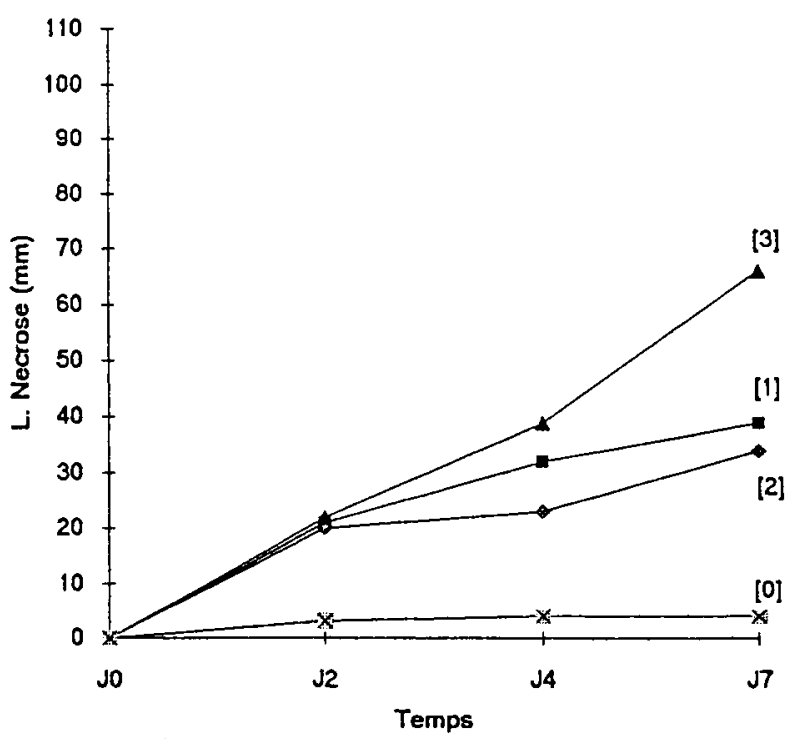

\section{SOUCHE 183}

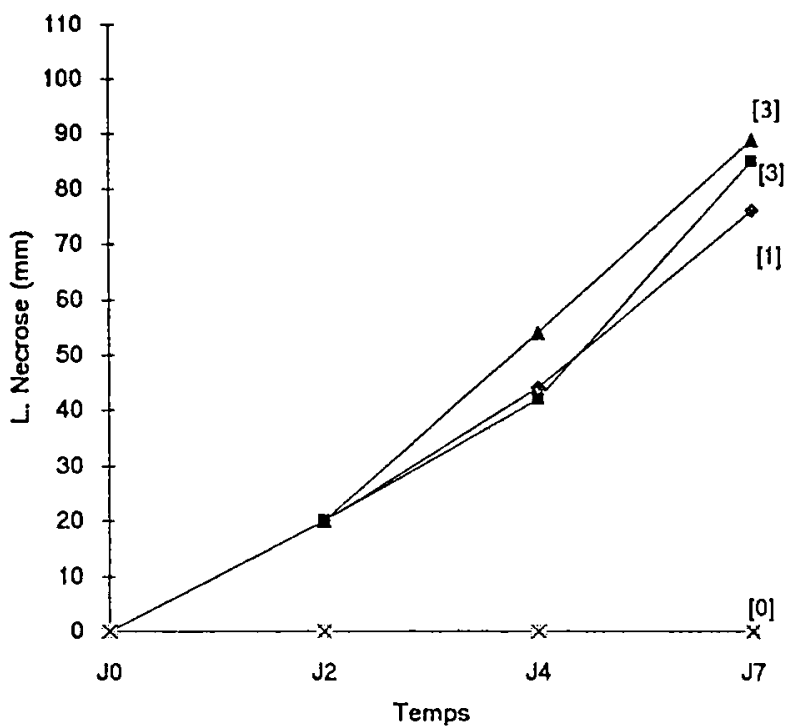

\section{SOUCHE 44}

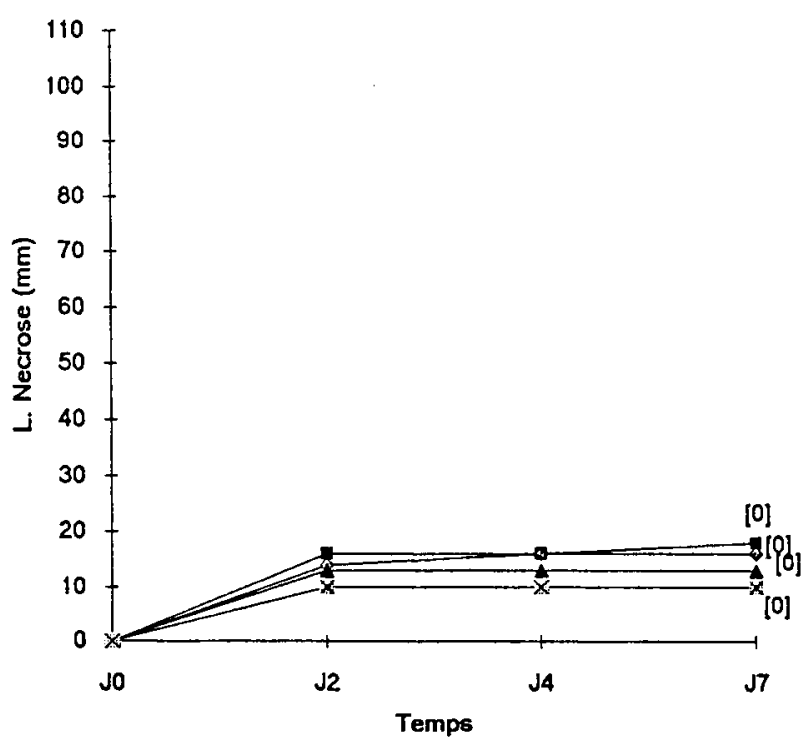

Fig 2. Envahissement caulinaire (en $\mathrm{mm}$ ) en fonction du temps, des 4 génotypes de tabac : Xanthi nc --49-10 --- ; 46-8 --- --- inoculés par les isolats compatibles; NIC 183, NIC 181, NIC 310 et incompatible : PAR 44. [x] = nombre de pétioles envahis à $\mathbf{J 7}$.

plus variable selon les génotypes et les essais considérés.

\section{Comportement de l'isolat NIC 310}

Cet isolat de black-shank peu agressif (fig 2) produit de la parasiticéine. Dans la figure 3 , nous avons tracé les courbes d'accumulation de la PR 1a dans la première feuille de tabac var Xanthi nc, après inoculation par NIC 181, NIC 310 et PAR 44. Dans l'interaction incompatible avec PAR 44, on note un pic d'accumulation $2,6 \mu \mathrm{g} /$ gMF à $\mathrm{J} 1$. La teneur diminue entre $\mathrm{J} 2$ et $\mathrm{J} 4$ pour augmenter à nouveau à $\mathrm{J} 7$.

Dans l'interaction compatible avec NIC 181, l'induction plus tardive à $\mathrm{J} 4$ marque le début du 


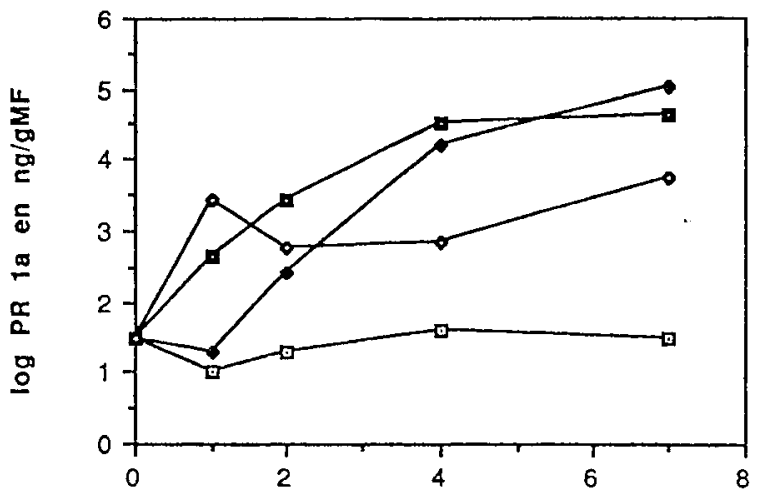

Fig 3. Cinétique d'accumulation de PR 1a dans la première feuille ( $F 1)$ de Xanthi nc, inoculé par les isolats compatibles NIC $181(E-)$-.- N.-. NIC $310\left(E_{+}\right)$-.compatible PAR $44(E+) \cdots \diamond \cdots--;$ témoin --- $\boxminus-\cdots ;$ valeur de $P R$ 1a en échelle logarithmique.

flétrissement du limbe foliaire. La teneur s'élève à $4 \mu \mathrm{g} / \mathrm{gMF}$ à $\mathrm{J} 4,21 \mu \mathrm{g} / \mathrm{gMF}$ à $\mathrm{J} 7$. Avec l'isolat compatible NIC 310 producteur d'élicitine, la teneur s'élève déjà à 2,6 $\mu \mathrm{g} / \mathrm{gMF}$, pour atteindre $33 \mu \mathrm{g} / \mathrm{gMF}$ à $\mathrm{J} 4$ et $45 \mu \mathrm{g} / \mathrm{gMF}$ à $\mathrm{J} 7$.

\section{DISCUSSION}

\section{Teneur en PR 1a des tabacs sains avant et après la décapitation (tableau II)}

Les feuilles de tabacs sains de la variété Xanthi nc renferment entre $0,01 \mu \mathrm{g} / \mathrm{gMF}$ et $0,37 \mu \mathrm{g} / \mathrm{gMF}$ de PR 1a : ces valeurs sont de l'ordre de celles citées dans la littérature (Abad et al, 1988, 1989). Les quantités dosées dans la variété Escambray sont nettement plus élevées : ces génotypes peuvent contenir de la PR 1a à l'état constitutif comme certains hybrides interspécifiques cités par Ahl et Gianinazzi, en 1982, ou bien leur stade physiologique, dans nos conditions d'essais, pouvait être différent de celui de la variété Xanthi nc. On sait que des protéines PR sont synthétisées au moment de la floraison chez les tabacs sains Xanthi nc (Fraser, 1981) : le décalage de précocité entre les 4 génotypes pourrait expliquer les différences observées.

L'effet "décapitation" n'entraîne pas, semble$\mathrm{t}$-il, de modification nette et importante dans le temps, de la teneur en PR 1a, si ce n'est tardivement à $\mathrm{J} 7$, dans la seconde feuille de la lignée 46-8. Van Loon (1983) rapporte qu'une blessure ne modifie pas cette teneur. L'accumulation tardive dans la lignée 46-8 pourrait peut-être marquer la reprise du bourgeonnement axillaire sur les plants décapités, quand on sait que les plants in vitro de Xanthi nc, ou Samsun, dont le bourgeonnement axillaire est stimulé par des cytokinines, en renferment des quantités élevées (Poupet et al, 1990).

\section{Expression de la résistance et accumulation de PR 1a}

Pour Kassanis (1981), l'accumulation des protéines $\mathrm{PR}$ est associée à l'état de résistance; pour Gianinazzi (1984), elles marquent l'hypersensibilité et la résistance acquise. D'après nos résultats, leur rôle n'apparaît pas aussi clairement. Leur teneur dans les tabacs sains n'est pas corrélée au niveau de résistance nonspécifique : ainsi, la variété Xanthi nc et la lignée 49-10, qui ont un même niveau de sensiblité aux différents isolats de Ppn, ont des teneurs en PR 1a qui varient dans un rapport de 1 à 100 .

Dans les interactions compatibles, l'envahissement du pétiole pour les 4 génotypes déclenche leur accumulation (tableau V). Pour De Witt et Bakker (1980) dans le couple tomate/ Cladosporium fulvum, la synthèse de protéines solubles, analogues aux protéines PR du tabac, accompagnerait le passage du champignon de la

Tableau IV. Accumulation de PR 1a en $\mu \mathrm{g} / \mathrm{gMF}$ dans l'interaction d'incompatibilité spécifique génotype R1/race r0.

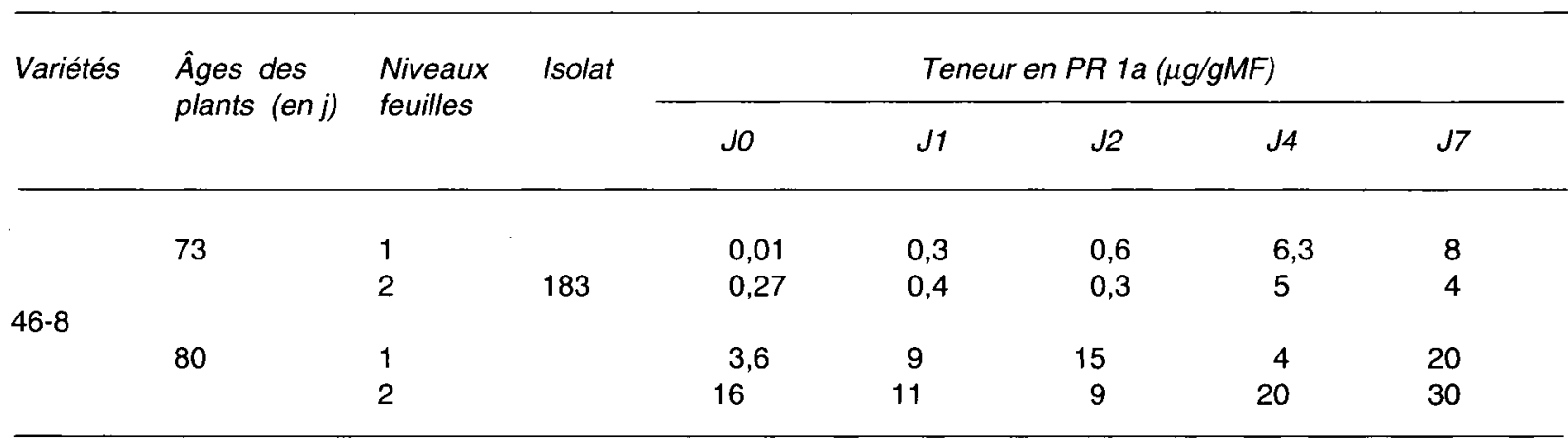


Tableau V. Accumulation de PR 1a en $\mu \mathrm{g} / \mathrm{gMF}$ dans la première feuille (F1) et la deuxième feuille (F2) dans les interactions compatibles.

\begin{tabular}{|c|c|c|c|c|c|c|c|c|}
\hline \multirow[t]{2}{*}{ Variétés } & \multirow{2}{*}{$\begin{array}{l}\text { Âges des } \\
\text { plants } \\
\text { (en j) }\end{array}$} & \multirow{2}{*}{$\begin{array}{l}\text { Niveau } \\
\text { de } \\
\text { feuilles }\end{array}$} & \multirow[t]{2}{*}{ Isolats } & \multicolumn{5}{|c|}{ Teneur en PR 1a $(\mu g / g M F)$} \\
\hline & & & & JO & $J 1$ & $J 2$ & $J 4$ & $J 7$ \\
\hline \multirow[t]{4}{*}{ Xanthi nc } & 60 & 1 & 181 & 0,03 & 0,02 & 0,2 & 16 & 108 \\
\hline & & 2 & & 0,01 & 0,03 & 0,1 & 3 & 43 \\
\hline & & 1 & 183 & 0,03 & 0,1 & 0,1 & 4 & 21 \\
\hline & & 2 & & 0,01 & 0,06 & 0,4 & 0,02 & 18 \\
\hline \multirow[t]{4}{*}{ Escambray } & 60 & 1 & 181 & 7 & 24 & 18 & 273 & 711 \\
\hline & & 2 & & 22 & 31 & 11 & 23 & 24 \\
\hline & & 1 & 183 & 7 & 4 & 18 & 66 & 260 \\
\hline & & 2 & & 22 & 7 & 4 & 17 & 19 \\
\hline \multirow[t]{4}{*}{$49-10$} & 73 & 1 & 181 & 0,8 & 4,6 & 1 & 44 & 403 \\
\hline & & 2 & & 1 & 1,6 & 3,4 & 49 & 436 \\
\hline & & 1 & 183 & 0,8 & 1,7 & 14 & 32 & 373 \\
\hline & & 2 & & 1 & 1,1 & 2 & 11 & 472 \\
\hline \multirow[t]{4}{*}{$46-8$} & 73 & 1 & 181 & 0,01 & 0,08 & 0,3 & 29 & 261 \\
\hline & & 2 & & 0,27 & 0,04 & 0,9 & 0,4 & 29 \\
\hline & 80 & 1 & 181 & 3,6 & 2,6 & 5 & 56 & 403 \\
\hline & & 2 & & 16,3 & 10 & 6 & 11 & 42 \\
\hline
\end{tabular}

biotrophie à la nécrotrophie. Chez le tabac, on a pu observer que les PR sont associées aux situations de stress (Hogue et al, 1987), ou induites par la plasmolyse (Wagih et Coutts, 1981) : le flétrissement du limbe peut être assimilé à un stress.

La résistance spécifique de l'interaction 46-8 (R1)/NIC 183 race ro n'entraîne aucun processus d'accumulation dans les feuilles 1 ou 2 (tableau IV) : cela peut s'expliquer par le fait que l'interface hôte-parasite est limité à un très faible nombre de cellules sans réaction nécrotrique systémique visible.

Leur accumulation dans les interactions d'«incompatibilité générale» est plus complexe à interpréter : en effet, elle varie avec le génotype de la plante-hôte et avec la souche incompatible, selon qu'elle est nécrosante ou non nécrosante. Dans la variété Xanthi nc, la souche PAR 44 induit dès J1 l'accumulation de PR 1a en l'absence de symptôme nécrotique visible (tableau III); les élicitines déclencheraient une accumulation précoce et directe de PR 1a du type de celle obtenue avec certains inducteurs chimiques (Asselin et al, 1985 ; Abad et al, 1989). Les 3 génotypes Escambray, 49-10, 46-8 ne répondent pas à cette induction. Leur absence de réactivité à la parasiticéine pourrait être expliquée par le bruit de fond dû au taux non négligeable de PR à l'état constitutif. Cependant, dans les lignées 49-10 et
46-8, inoculées par CRY 52 très nécrosante, le taux de PR 1a s'est accrue fortement dès J4 (tableau III) ; cette accumulation plus tardive serait étroitement associée au phénomène nécrotique déjà décrit par Bonnet et al (1986).

Dans les interactions tabac-Phytophthora étudiées, l'accumulation de PR 1a aurait plusieurs modes d'induction : précoce, dès $\mathrm{J} 1$, en réponse à l'élicitine, plus tardif à $\mathrm{J4}$, marquant soit l'envahissement des tissus dans les interactions compatibles, soit les nécroses à distance de l'incompatibilité générale. L'analyse comparée des cinétiques d'accumulation dans la variété Xanthi nc inoculée par NIC 181, non productrice d'élicitine (E-) ou NIC 310 et PAR 44 productrices $(E+)$, répond à ce schéma (fig 3 ). Avec les 2 isolats $(E+)$, le taux s'élève dès $J 1$, il cesse d'augmenter avec l'isolat incompatible PAR 44 qui n'envahit pas la tige. Avec l'isolat compatible NIC 310 qui colonise le pétiole, il continue de croître. Avec l'isolat compatible NIC 181 (E-), l'augmentation plus tardive de PR 1a marque seulement l'envahissement du pétiole.

Toute réaction nécrotique à distance ou envahissement des limbes foliaires s'accompagnent d'une augmentation de PR 1a, mais nous n'avons pu mettre en évidence de relation directe entre la résistance et la synthèse de PR 1a. 


\section{RÉFÉRENCES}

Abad P (1986) Aspects moléculaires des mécanismes de résistance chez Nicotiana tabacum en réponse à des agressions induites par des agents pathogènes ou abiotiques. Thèse de doctorat sciences, université Paris Sud, Orsay, $140 p$

Abad P, Marais A, Cardin L, Poupet A, Ponchet M (1988) The effect of benzoic acid derivatives on Nicotiana tabacum growth in relation to PR-b1 production. Antiviral Res 9, 315-327

Abad P, Cardin L, Poupet A, Ponchet M (1989) Comparison of pathogenesis related «b1» protein determination obtained by Elisa and HPLC techniques. J Phytopathol 124, 175-188

Ahl P, Gianinazzi S (1982) b protein as a constitutive component in highly (TMV) resistant interspecific hybrids of Nicotiana glutinosa $\times$ Nicotina debneyi. Plant Sci Lett 26, 173-181

Asselin A, Grenier J, Cote F (1985) Light influenced extracellular accumulation of $b$ (pathogenesisrelated) proteins in Nicotiana green tissue induced by various chemicals or prolonged floating on water. Can J Bot 63, 1276-1283

Bonnet P, Poupet A, Abad P, Venard P, Cardin L (1986) Induction de nécroses foliaires, de protéines b et de résistance dans les interactions tabac/ Phytophthora. agronomie 6, 829-837

Cardin L, Salle D, Poupet A, Ponchet M (1983) Dosage immunoenzymatique du virus de la mosaïque du tabac dans différents tissus cultivés in vitro. agronomie 3, 983-988

De Witt PJ, Bakker J (1980) Differential changes in soluble tomato leaf proteins after inoculation with virulent and avirulent race of Cladosporium fulvum (syn Fulvia fulva). Physiol Plant Pathol 17, 121-130

Fraser RS (1981) Evidence for the occurrence of the «pathogenesis-related» proteins in leaves of healthy tobacco plants during flowering. Physiol Plant Pathol 19, 69-76

Gianinazzi S (1984) Genetic and molecular aspects of resistance induced by infection or chemicals. In: Plant-microbe interactions: molecular and genetic perspective (Kosuge T, Nester EW, eds) Macmillan Publishing Co, New York, 321-352

Heath MC (1981) A generalized concept of host parasite specificity. Phytopathology 71, 1121-1123

Helgeson JP, Haberlach GT, Upper CD (1976) A dominant gene conferring disease resistance to tobacco plants expressed in tissue cultures. Phytopathology 66, 91-96

Hogue R, Asselin A (1987) Detection of 10 additional pathogenesis related (b) proteins in intracellular fluid extracts from stressed Xanthi nc tobacco leaf tissue. Can J Bot 65, 476-481

Kassanis B (1981) Some speculations on the nature of the natural defense machanism of plants against virus infection. Phytopathol $Z 102,277-291$

Kauffmann S (1988) Les protéines PR (pathogenesisrelated) du tabac. Des proteines impliquées dans les réactions de défense aux agents phytopathogènes. Isolement, propriétés sérologiques et activités biologiques. Thèse doctorat biologie moléculaire, université Louis-Pasteur, Strasbourg I, $85 \mathrm{p}$

Poupet A, Cardin L, Bettachini B, Beck D (1990) Accumulation de protéines solubles (PR 1a protéine) dans des bourgeons de Nicotiana tabacum cultivés in vitro en présence de cytokinine. CR Acad Sci Sér III 311, 239-146

Ricci $P$, Bonnet $P$, Huet JC, Sallantin $M$, BeauvaisCante F, Bruneteau M, Billard V, Michel G, Pernollet JC (1989) Structure and activity of proteins from pathogenic fungi Phytophthora eliciting necrosis and acquired resistance in tobacco. Eur J Biochem $183,55-563$

Stintzi A, Heitz T, Kauffmann S, Geoffroy P, Legrand M, Fritig B (1990) Protéines de défense du tabac. $2^{\mathrm{e}}$ congrès de la Société française de phytopathologie, Strasbourg, $1 \mathrm{p}$

Van Loon LC (1983) Mechanisms of resistance in virus-infected plants. In: The Dynamics of Host Defense (Bailey JA, Deverall BJ, eds) Academic Press, Sydney, Australia, 123-190

Wagih EE, Coutts RH (1983) Similarities in the soluble protein profiles of leaf tissue following either a hypersensitive reaction to virus infection or plasmolysis. Plant Sci Lett 21, 61-69 